\title{
Theory of Carbon Nanotubes as Optical Nano Waveguides
}

\author{
Afshin Moradi ${ }^{1,2}$ \\ ${ }^{1}$ Department of Nano Science, Kermanshah University of Technology, Kermanshah, Iran; ${ }^{2}$ Department of Nano Science, Institute for \\ Studies in Theoretical Physics and Mathematics (IPM), Tehran, Iran \\ E-mail: a.moradi@nano.ipm.ac.ir
}

Received November $5^{\text {th }}, 2010$; revised November $25^{\text {th }}, 2010$; accepted December $1^{\text {st }}, 2010$.

\begin{abstract}
The propagation of surface plasmon waves in metallic single-walled carbon nanotubes is analyzed within the framework of the classical electrodynamics. The conduction electrons of the system are modelled by an infinitesimally thin layer of free-electron gas which is described by means of the semiclassical kinetic theory of the electron dynamics. The effects of the energy band structure is taken into account and a more accurate dispersion relation for surface plasmon oscillations in the zig-zag and armchair nanotubes of metallic character is obtained.
\end{abstract}

Keywords: Carbon Nanotubes, Dispersion Relation, Plasmon Oscillations

\section{Introduction}

With the discovery by Iijima [1] of carbon nanotubes (CNTs) structures, a new class of materials with a reduced dimensionality has been introduced. Metallic CNTs are considered suitable candidates in the field of plasmonics as new plasmonics waveguides [2-12]. These new plasmonic waveguides can be built by some simple and well-known methods such as CVD [12].

By using the classical electrodynamics and a semiclassical kinetic theory, Slepyan et al [2], derived the dispersion relation of surface waves in single-walled carbon nanotubes (SWCNTs) and for the first time, found that CNTs can be used as a nano waveguide for controlling electro magnetic wave propagation in specified frequency ranges (for instance, infrared and optical). In particular, by solving Maxwell and hydrodynamic equations, the propagation of electromagnetic waves in SWCNTs is studied in Reference [4,5] and it has been shown that dispersion behaviors of the plasma waves with TM and TE modes are quite similar. However, the hydrodynamic theory, without any effects of the energy band structure to be taken into account, can not be valid enough for the investigations of plasmon waves propagation in CNTs.

SWCNTs are quasi one-dimensional material, which could be regarded as a rolled-up graphene layer (i.e., a mono-atomic layer of graphite) in the cylindrical form. It has a radius of a few nanometers and lengths up to centimeters. A graphene layer is a semi-metallic material. Nevertheless, when a graphene layer is rolled up it may become either metallic or semiconducting, depending on its geometry. Figure 1 shows a graphene layer. The geometric structure of a SWCNT is uniquely determined by the chiral vector $\mathrm{R}=m a_{1}+n a_{2} \equiv(m, n)$, where $\mathrm{m}$ and $\mathrm{n}$ are integers, and $\mathrm{a} 1$ and $\mathrm{a} 2$ are the elementary vectors of the dimensional graphene lattice (see Figure 1). The tube radius of the CNTs is given by

$$
r_{c}=\frac{a_{0}}{2 \pi} \sqrt{m^{2}+m n+n^{2}},
$$

where $a_{0}=\sqrt{3} b_{0}$ is the lattice constant of the graphite sheet and $b_{0}=1.42^{\circ} \mathrm{A}$ is the distance between the nearest-neighboring carbon atoms. A SWCNTs is metallic if $|m-n|=3 q$, where $q=0,1,2, \cdots$ Thus, armchair nanotubes are always metallic, whereas zig-zag nanotubes are metallic only if $m=3 q$ with $q=1,2, \cdots$

In this Letter, we study the energy band effects on the dispersion relation of the surface plasmon waves in SWCNTs of metallic character, by using the semiclassical kinetic theory of the electron dynamics. In comparison with previous investigations $[4,5]$ that focus on plasmon wave oscillations in a cylindrical electron gas as a simple model of metallic tubes, present work stresses on more exact analysis of geometrical effects, including the radius and the chiral angle of the nanotube. 


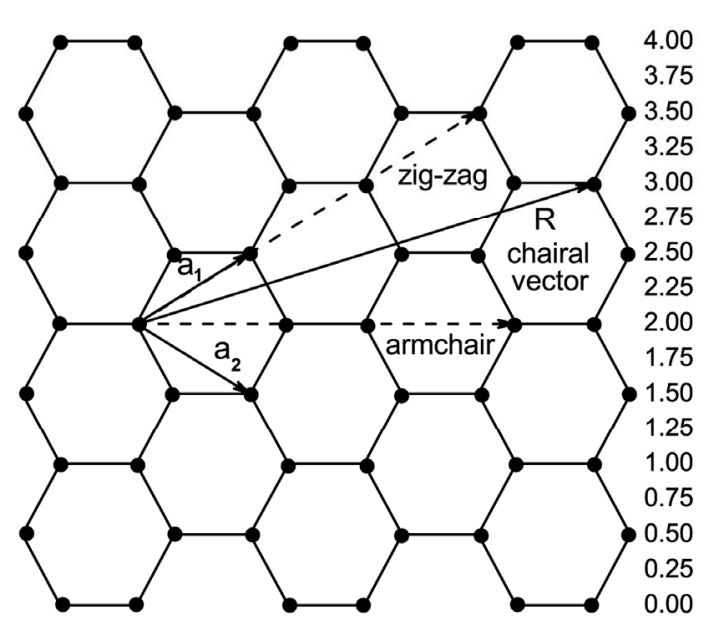

Figure 1. Graphene sheet used in forming CNTs (The dots illustrate the carbon atoms positions). The lattice basis vectors are a1 and a2. The chiral vector $\mathrm{R}=m a_{1}+n a_{2} \equiv$ $(m, n)$, where $\mathbf{m}$ and $\mathbf{n}$ are integers. The nanotubes with $n=0$ are called zig-zag, those with $m=n$ are called armchair and those with $m \neq n$ are called chiral.

\section{Formulation of the Problem}

Let us consider both zig-zag $(m, 0)$ and armchair $(m, m)$ nanotubes as infinitesimally thin and infinitely long cylindrical shells of radius $r_{c}$ with its axis along the $z$-direction and regard the CNT to consist of $\pi$-electrons superimposed with equilibrium densities (per unit area) $n_{0}$. We assume that in equilibrium the $\pi$-electron fluid has no velocity and $n$ is the perturbed density (per unit area) of fluid, produced by the $\pi$-electrons themselves under the action of the electric field generated by the fixed positive ions of the lattice. Hydrodynamic theory describes electronic motion in terms of two dynamical variables, namely the electrondensity fluctuation, $n(x, t)$ and $u(x, t)=\left(u_{\phi}, u_{z}\right)$ The basic equations in this linearized hydrody- namic model are the equations of motion, the equations of continuity,

$$
\begin{gathered}
\frac{\partial n(x, t)}{\partial t}=-n_{0} \nabla_{\|} \cdot u(x, t), \\
n_{0} \frac{\partial u(x, t)}{\partial t}=-\alpha \nabla_{\|} n(x, t)-e \frac{n_{0}}{m_{e f f}} E_{\|}(x, t)-\gamma n_{0} u(x, t),
\end{gathered}
$$

where $E_{\|}(x, t)=E_{z} \hat{e}_{z}+E_{\phi} \hat{e}_{\phi}$ is the tangential component of the electromagnetic field, $e$ is the element charge, $m_{\text {eff }}$ is the effective mass of the $\pi$-electrons and $\nabla_{\|}=e_{z}(\partial / \partial z)+a^{-1} e_{\phi}(\partial / \partial \phi)$ differentiates only tangentially to the nanotubes surface. In the right-hand side of Equation (2), the first term arise from the internal interaction force in the fluid with $\alpha=v_{F}^{2} / 2$ that is the square of the speed of propagation of density disturbances in a uniform 2D homogeneous electron fluid. The second term is the force on $\pi$-electron fluid due to the tangential component of the electric field, evaluated at the nanotube surface $r=r_{c}$ and the last term represents the effects of the scattering of the electrons with the positive-charge background, where $\gamma$ being the friction coefficient. We note that this term has been neglected in the previous works [4,5].

Let us deal with the surface plasmon waves with TM modes only in this paper, the similar result for TE mode can be obtained. The electric field vector $E(x, t)$ can be expanded in the following Fourier forms

$$
E(x, t)=\sum_{m=-\infty}^{+\infty} \int_{-\infty}^{+\infty} d q E_{m}(r, q) \exp [i(m \varphi+q z-\omega t)] .
$$

For the TM modes, the field components can be expressed in terms of $E_{z m}$, and it is readily shown that this satisfy

$$
\frac{1}{r} \frac{d}{d r}\left(r \frac{d}{d r} E_{z m}\right)-\left(\kappa^{2}+\frac{m^{2}}{r^{2}}\right) E_{z m}=0,
$$

where $\kappa^{2}=q^{2}-\omega^{2} / c^{2}$ and $c$ is the light speed. The parameter $\kappa$ is a real quantity in the region

$$
\frac{\omega}{q}<c
$$

This means that we deal with the slow transverse magnetic waves. By eliminating the velocity field $u(x, t)$, one can obtain the following equation from Equations (1) and (2)

$$
\left(\frac{\partial^{2}}{\partial t^{2}}+\gamma \frac{\partial}{\partial t}\right) n(x, t)=\alpha \nabla_{\|}^{2} n(x, t)+e \frac{n_{0}}{m_{e f f}} \nabla_{\|} \cdot E_{\|}(x, t) .
$$

Upon solving Equation. (6) by means of the space-time Fourier transforms for the induced density $n(x, t)$ on the cylindrical surface, we find

$$
n(x, t)=\sum_{m=-\infty}^{+\infty} \int_{-\infty}^{+\infty} d q N_{m}(q) \exp [i(m \phi+q z-\omega t)],
$$

where

$$
N_{m}=-\frac{i e}{\Omega_{m}} \frac{n_{0}}{m_{e f f}}\left(q E_{z}+\frac{m}{r_{c}} E_{\phi}\right),
$$

and $\Omega_{m}=(\omega+i \gamma) \omega-\alpha\left(q^{2}+m^{2} / r_{c}^{2}\right)$. Now, we use the appropriate boundary condition, we have

$$
\left.E_{r m}\left(r_{c}\right)\right|_{r>r_{c}}-\left.E_{r m}\left(r_{c}\right)\right|_{r<r_{c}}=-\frac{e}{\varepsilon_{0}} N_{m}
$$


where $\varepsilon_{0}$ is the permittivity of free space and the radial component $E_{r m}$ and the azimuthal component $E_{\phi m}$ of the electric field, are given by

$$
E_{r m}(r)=-i \frac{q}{\kappa^{2}} \frac{d E_{z m}(r)}{d r},
$$

and

$$
E_{\phi m}(r)=\frac{m q}{\kappa^{2} r} E_{z m}(r) .
$$

On the other hand, the relevant solution of Equation (4) is

$$
E_{z m}(r)=E_{0 z} K_{m}\left(\kappa r_{c}\right) I_{m}(\kappa r) \quad\left(r<r_{c}\right),
$$

and

$$
E_{z m}(r)=E_{0 z} I_{m}\left(\kappa r_{c}\right) K_{m}(\kappa r)\left(r>r_{c}\right),
$$

where $I_{m}(x)$ and $K_{m}(x)$ are the modified Bessel functions. Substituting Equations (12-13) into boundary condition Equation (9), by using Equation (8), for $q \gg \omega / c$, one can obtain the dispersion equation as below:

$$
(\omega+i \gamma) \omega=\alpha\left(\kappa^{2}+\frac{m^{2}}{r_{c}^{2}}\right)+\omega_{p}^{2} r_{c}^{2}\left(\kappa^{2}+\frac{m^{2}}{r_{c}^{2}}\right) I_{m}\left(\kappa r_{c}\right) K_{m}\left(\kappa r_{c}\right),
$$

where $\omega_{p}=\left(e^{2} n_{0} / \varepsilon_{0} r_{c} m_{\text {eff }}\right)^{1 / 2}$ is the eigen-frequency of the $\pi$-electron gas layer in metallic SWCNTs. The solutions of Equation (14) yield complex frequencies $\omega=\omega_{r}+i \omega_{i}$. It may be observed that the imaginary part $\omega_{i}$ will be given simply by $-\gamma / 2$. In fact, by writing $\omega=\omega_{r}-i \gamma / 2$, the solutions for finite damping will be of the form

$$
\omega=\left\{\alpha\left(\kappa^{2}+\frac{m^{2}}{r_{c}^{2}}\right)+\omega_{p}^{2} r_{c}^{2}\left(\kappa^{2}+\frac{m^{2}}{r_{c}^{2}}\right) I_{m}\left(\kappa r_{c}\right) K_{m}\left(\kappa r_{c}\right)-\frac{\gamma^{2}}{4}\right\}^{1 / 2}-i \frac{\gamma}{2} .
$$

The friction coefficient is the inverse of the electron relaxation time $\tau$. For CNTs it is taken as $\tau=$ $3 \times 10^{-12} s$ [13], so we have $\gamma=1 / 3 \times 10^{12} \mathrm{~Hz}$. Formally speaking, the dispersion characteristics of the surface waves in the system is dependent on the nanotube geometry (including the radius and the chiral angle of the nanotube), the wave number, the angular momentum, and the friction coefficient. However, it is easy to find that by increasing friction coefficient, the dispersion curves shift to lower frequencies, so in the following we set $\gamma=0$.

At this stage, from Equation (15), one can see for investigation the dispersion characteristics of the zig-zag and armchair SWCNTs, we have to give the values of $n_{0} / m_{\text {eff }}$. The parameter $n_{0} / m_{\text {eff }}$ takes into account the influence of the atomic crystal field. By using the semiclassical model of the $\pi$-electron dynamics, Miano and Villone [14], obtained the following estimation:

$$
\frac{n_{0}}{m_{\text {eff }}} \cong \frac{2 v_{F}}{\pi^{2} \hbar} \frac{1}{r_{c}},
$$

where $v_{F}$ is the velocity of the electrons at the Fermi level

$$
v_{F}=\frac{3 \gamma_{0} b_{0}}{2 \hbar} .
$$

$\gamma_{0}$ is a characteristic energy $\left(\gamma_{0} \sim 2.7-3 e \mathrm{~V}\right)$ of the graphene lattice and $\hbar$ is the Planck constant; it results that $v_{F} \sim(0.9-1) \times 10^{6} \mathrm{~m} / \mathrm{s}$. The Equation (16) holds for zig-zag nanotubes with $m=3 q<60$, for armchair nanotubes with $m<50$ and for chiral nanotubes with $2 n+m=3 q$. In the range of validity of Equation (16), the parameter decreases as the nanotube radius increases.

To see clearly the energy band effects on the dispersion relation of the surface waves in SWCNTs, in the following we consider long and short wavelength limits of the Equation (15). For $\kappa r_{c} \rightarrow \infty$, by using the well-known asymptotic expressions [15],

$I_{m}(x)=\frac{e^{x}}{\sqrt{2 \pi x}}$ and $K_{m}(x)=\sqrt{\frac{\pi}{2 x}} e^{-x} \quad$ (with the finite $m$ ), the dispersion relation can be written approximately as

$$
\omega^{2}=\alpha \kappa^{2}+\frac{e^{2} v_{F}}{\varepsilon_{0} \pi^{2} \hbar} \frac{\kappa}{r_{c}} .
$$

One can see unlike the case of Equation (3) in Ref. 5, where the dispersion relation is independent of the geometrical effects of the tube, the right-hand side of Equation (17) in the present work, depends strongly on the radius of the nanotube. It is easy to find that as the values of the nanotube radius $r_{c}$ increases the values of $\omega$ decrease.

In the opposite limit $\kappa r_{c} \rightarrow 0$, where the phase velocity of the surface plasmon is comparable to the velocity of light, surface plasmon oscillations couple with the electromagnetic wave and retardation effects are present. Retardation effects on low-dimensional plasmons are investigated in details in Reference [16]. If we neglect the retardation effects, by using the well-known expressions of Bessel functions,

$$
I_{m}(x)=\frac{1}{\Gamma(m+1)}\left(\frac{x}{2}\right)^{m},
$$

$K_{m}(x)=\frac{\Gamma(m)}{2}\left(\frac{2}{x}\right)^{m} \quad($ for $m \neq 0)$ and $K_{0}(x)=\frac{\ln 1.123}{x}($ for $m=0)$ then we may obtain for $m=0$

$$
\omega(m=0, \kappa \approx 0) \approx\left[\frac{4 e^{2} v_{F}}{\varepsilon_{0} \pi^{2} \hbar} \ln \left(\frac{1.123}{\kappa r_{c}}\right)\right]^{1 / 2} \kappa,
$$


that is a quasiacoustic mode and for $m \neq 0$, we get

$$
\omega^{2}=\frac{\alpha}{r_{c}^{2}} m^{2}+\frac{e^{2} v_{F}}{\varepsilon_{0} \pi^{2} \hbar r_{c}^{2}} m .
$$

which is also quite sensitive to the geometric of the nanotube. Comparing the long-wavelength and short-wavelength limits, it can be seen that the energy band structure play an important role in the dispersion relation, for all values of wavelength.

To better understand the energy band effects on the dispersion relation of the plasmon waves in the system, we illustrate in Figure 2, the dependence of the frequency $\omega$ on the variable $q$ for different nanotube geometries with $m=0$ and 1 . One can see that the dispersion curves $\omega$ for the zig-zag $(27,0)$ with radius $r_{c}=1.056 \mathrm{~nm}$ and armchair $(15,15)$ with radius $r_{c}=$ $1.017 \mathrm{~nm}$ are largely similar. This means that behaviors of the plasmon waves are not sensitive to the types of metallic nanotubes with same radius.

In order to study the mechanism of exciting plasmon waves on the SWCNTs for future waveguide usage, we plot the speed lines of three electron beams in Figure 2(a), by considering the expression $\omega=v q$. As seen, when the electron beam velocities locate in the range $0.93 \times 10^{6} \mathrm{~m} / \mathrm{s}<v<4 \times 10^{6} \mathrm{~m} / \mathrm{sv}$ (i.e., the velocity of the electron beam can be equal to the phase velocity of the surface plasmon modes), the electron beam is in synchronization with the surface waves, and they interact with each other and instability occurs between them. Thus, we conclude that surface waves in the system can only be excited by applying some relativistic electron beams with the speed of about $10^{6} \mathrm{~m} / \mathrm{s}$.

Finally, let us look at the spatial extension of the electromagnetic field associated with the surfaceplasmon polariton as shown in Figure 3, for a nanotube with radii $r_{c}$. The attenuation length is determined at long- wavelengths (where retardation effects are present) by means of the penetration depth. However in the nonretarded surface plasmon condition, i.e., $q \gg \omega / c$, the penetration depth in the system is $\ell \sim 1 / q$ thereby leading to a strong concentration of the electromagnetic surface-plasmon field near the interface. This means that the dispersion curves in Figures 2 and 3, can show the relation between frequency and the inversion penetration depth. From Figures 2(a) and 2(b), one can see that, as increasing the wave frequency, the radial penetration depth of the TM surface modes decrease

\section{Conclusions}

In summary, a theoretical model based on the classical electrodynamics and linearized hydrodynamic theory is employed to describe the plasmon wave propagation on the surface of the metallic SWCNTs, where the effects of the energy band structure is taken into account. It has been found that the nanotube geometry play an important

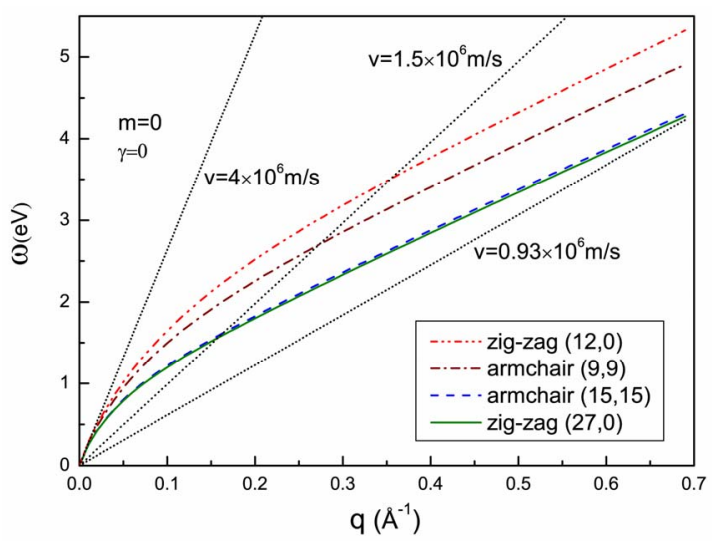

(a)

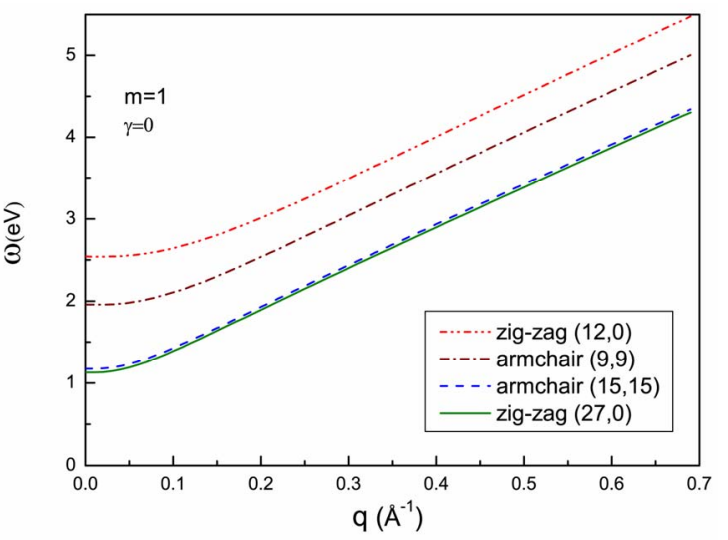

(b)

Figure 2. The dispersion curves from the surface waves for different nanotube geometries, for (a) $m=0$ and (b) $m=1$, respectively. The straight dotted lines correspond to different $v$ for $\omega=v q$.

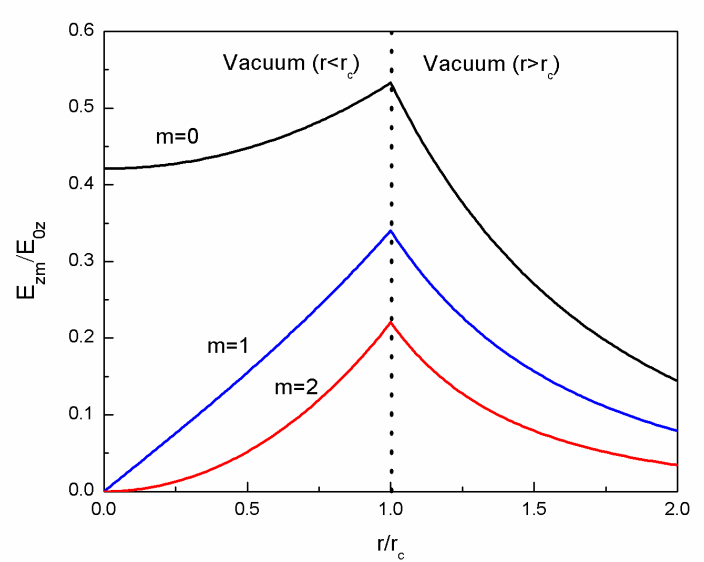

Figure 3. Surface TM modes of a nanotube as a function of the radial coordinate $r$. 
role in the dispersion relation of the surface waves, for all value of wavelength. Also, numerical results show that behaviors of the plasmon waves are not sensitive to the types of metallic nanotubes with same radius. In addition, the results obtained make us believe that the hydrodynamic theory in conjunction with semiclassical model is available and appropriates for studies of the plasmon wave oscillations in CNTs, especially for different nanotube geometries.

\section{REFERENCES}

[1] S. Iijima, "Helical Microtubules of Graphitic Carbon," Nature, Vol. 354, No. 6348, 1991, pp. 56-58.

[2] G. Ya. Slepyan, S. A. Maksimenko, A. Lakhtakia, O. M. Yevtushenko and A. V. Gusakov, "Electronic and Electromagnetic Properties of Nanotubes," Physical Review B, Vol. 57, 1998, pp. 9485-9497.

[3] M. V. Shuba, S. A. Maksimenko and A. Lakhtakia, "Electromagnetic Wave Propagation in an Almost Circular Bundle of Closely Packed Metallic Carbon Nanotubes,” Physics Review B, Vol. 76, No. 15, 2007, pp. 1-9.

[4] L. Wei and Y. N. Wang, "Electromagnetic Wave Propagation in Single-Wall Carbon Nanotubes," Physics Letters A, Vol. 333, 2004, pp. 303-309.

[5] H. Khosravi and A. Moradi, "Comment on: Electromagnetic Wave Propagation in Single-Wall Carbon Nanotubes,” Physical Letters A, Vol. 364, 2007, pp. 515-516.

[6] A. Moradi, "Guided Dispersion Characteristics of Metallic Single-Walled Carbon Nanotubes in the Presence of Dielectric Media,” Optics Communications, Vol. 283, No. 1, 2010, pp. 160-163.

[7] D. J. Mowbray, Z. L. Miskovic, F. O. Goodman and Y.-N. Wang, "Interactions of Fast Ions with Carbon Nanotubes:
Two-Fluid Model,” Physical Review B, Vol. 70, No. 19, 2004, pp. 1-7.

[8] D. J. Mowbray, S. Segui, J. Gervasoni, Z. L. Miskovi, and N. R. Arista, "Plasmon Excitations on a Single-Wall Carbon Nanotube by External Charges: TwoDimensional Two-Fluid Hydrodynamic Model,” Physical Review B, Vol. 82, No. 3, 2010, pp. 1-14.

[9] C. Javaherian and B. Shokri, "Guided Dispersion Characteristics of Metallic Single-Wall Carbon Nanotubes,” Journal of Physics D, Vol. 42, No. 5, 2009, pp. 1-6.

[10] L. Liu, Z. Han and S. He, "Novel Surface Plasmon Waveguide for High Integration,” Optics Express, Vol. 13, No. 17, 2005, pp. 6645-6650.

[11] E. Ozbay, "Plasmonics: Merging Photonics and Electronics at Nanoscale Dimensions,” Science, Vol. 311, No. 5758, 2006, pp. 189-193.

[12] M. Su, B. Zheng and J. Liu, "A Scalable CVD Method for the Synthesis of Single-Walled Carbon Nanotubes with High Catalyst Productivity," Chemical Physics Letters, Vol. 322, No. 5, 2000, pp. 321-326.

[13] Y. Miyamoto, S. G. Louie and M. L. Cohen, "Chiral Conductivities of Nanotubes," Physical Review Letteras, Vol. 76, No. 12, 1996, pp. 2121-2124.

[14] G. Miano and F. Villone, “An Integral Formulation for the Electrodynamics of Metallic Carbon Nanotubes Based on a Fluid Model," IEEE Transaction on Antennas and Propagation, Vol. 54, No. 10, 2006, pp. 2713-2724.

[15] M. Abramowitz and I. A. Stegum, Handbook of Mathematical Funcyions, Dover, New York, 1965.

[16] L. Wendler and T. Kraft, "Retardation Effects on Intraand Intersubband Plasmons in Quantum Wells and Their Manifestations in Grating-Coupler-Assisted Optical Transmission,” Physical Review B, Vol. 60, No. 24, 1999, pp. 16603-16610. 